\title{
A FIRST-ORDER SEQUENTIAL PREDICTOR-CORRECTOR REGULARIZATION METHOD FOR ILL-POSED VOLTERRA EQUATIONS*
}

\author{
WOLFGANG RING ${ }^{\dagger}$
}

\begin{abstract}
A discrete sequential predictor-corrector regularization method for the solution of ill-posed Volterra equations based on affine continuation into the future interval is presented. An infinite dimensional analogue is derived and analyzed. Stability estimates and convergence in the cases of exact and noisy data are proved. A convergence rate result is proved for finitely smoothing kernels if the exact solution is $\mathcal{C}^{1, \alpha}$-smooth. Numerical examples are presented. The results are compared to other standard regularization methods.
\end{abstract}

Key words. ill-posed Volterra equations, sequential regularization, Beck's method, first-order approximation, stability estimates, convergence rates

AMS subject classifications. 45D05, 45Q05, 47A52, 65F22, 65J20, 65R32

PII. S0036142999362092

1. Introduction. In this work we consider a sequential predictor-corrector regularization method for the solution of first-kind Volterra equations of the form

$$
\int_{0}^{t} k(t-s) u(s) d s=f(t)
$$

on $[0,1]$. The solution of a Volterra equation at time $t$ depends only on the data $f(s)$ for $s \leq t$. Because of this property numerical approaches to the solution of Volterra equations are usually sequential, which means that if we consider a finite number of time-steps $t_{0}, \ldots, t_{N}$, the solution at time $t_{i}$ is constructed from the current data value $f\left(t_{i}\right)$ and (already known) values of the solution at times $t_{j}$ with $j<i$. In a somewhat sloppy use of language we call a numerical scheme sequential if the solution $u\left(t_{i}\right)$ depends only on information from the past, i.e., from times $t_{j}$ with $j \leq i$.

The compactness of the Volterra operator (1.1) leads to a discontinuous dependence of the solution on the data. Therefore, ad hoc numerical methods (either sequential or not) produce highly unstable numerical results and some form of regularization must be introduced to obtain a numerically stable approximation scheme.

In this paper we consider a regularization method for Volterra equations which uses information also from future data points to obtain stable numerical results. Moreover, the method is sequential and therefore fast and suitable for online, real-time computations. This structure preserving property of the regularization scheme distinguishes it from many established regularization methods; for example, Tikhonov regularization would use information of the data over the whole time-interval $[0,1]$ to construct the solution at some intermediate time $0<t<1$. Moreover, a discretization of Tikhonov's method usually gives full matrices which cannot be solved sequentially.

Sequential regularization methods for ill-posed Volterra equations using information from the past and from a few future data points (in contrast to using information

\footnotetext{
* Received by the editors September 21, 1999; accepted for publication (in revised form) September 19, 2000; published electronically February 2, 2001. The research of this paper was partially supported by the Special Research Center on Optimization and Control at the University of Graz.

http://www.siam.org/journals/sinum/38-6/36209.html

†Special Research Center on Optimization and Control, University of Graz, Heinrichstrasse 36, A-8010 Graz, Austria (wolfgang.ring@kfunigraz.ac.at).
} 
over the whole time-domain) were introduced and investigated by Beck already in the early 1960s. A discrete version of the method we are considering can be found in Beck, Blackwell, and St. Clair [1, p. 131] under the name of "sequential linear function specification method."

More recently, Lamm made considerable contributions to the development and understanding of sequential regularization methods. Specifically with [4], [6], and [5] it could be shown that Beck's sequential constant function specification method can be interpreted as discretization of a properly defined Volterra equation of the second kind. Furthermore, it can be shown that the second-kind equation is solvable in a stable way [6]. It has also been shown that, as the data noise goes to zero, solutions of the approximating second-kind Volterra equations converge to the exact solution for finitely smoothing kernels under a certain stability condition (see [6]). For 1smoothing kernels, a convergence rate of order $O\left(\delta^{\frac{1}{2}}\right)$ was derived in [5]. Convergence rates for finitely smoothing kernels were recently proved in [10]. Several generalizations were considered in [9], [8], and [3]. We also refer to the survey article [7] and the numerous references cited therein for an overview of the literature on stable numerical methods for the sequential solution of ill-posed Volterra equations. We should also mention that the class of regularization methods under consideration here occurs under various names in the literature such as "local regularization," "predictor-corrector regularization," "future-sequential regularization," "Beck's method," or combinations of these notions.

The idea underlying Beck's method and its variants in the discrete case is to choose the value of the solution $u_{i}$ on the time-interval $\left[t_{i-1}, t_{i}\right]$ in such a way that a constant continuation of the solution with this value fits the given data not only for the current time $t_{i}$ but also, in a least-squares sense, at a few time-steps $t_{i+j}, j=1, \ldots, r$, in the future. In this paper we investigate a variant of Beck's method where we replace constant continuation by affine continuation. We refer to our approach as a "firstorder sequential regularization method" in contrast to the "zero-order method" based on constant continuation. In the infinite dimensional limit our method leads to a Volterra integro-differential equation of the second kind.

A similar generalization is presented in [3], where a polynomial of degree $d \geq 0$ is used for the continuation into the future interval. The structural difference between this approach and ours is that this polynomial does not connect continuously to the already determined part of the solution. Numerically our first-order method produces smoother results that the polynomial method in [3]. (See Figure 5.2 in section 5.)

In section 2 we derive the regularization scheme for the discrete case and generalize the discrete approach to find a second-kind Volterra integro-differential equation representing the regularization method in infinite dimensional space. Section 3 is devoted to the convergence analysis of the infinite dimensional regularization method. We obtain stability estimates and a convergence result in the case of exact data. For the class of finitely smoothing kernels we can also prove a convergence rate result. As usual in inverse problems the rate of convergence is determined by smoothness properties of the exact solution (Theorem 4.2). Here we could avoid imposing strong smoothness conditions on the exact solution to get convergence, as it is necessary in the analysis in [6]. The last section contains numerical experiments and comparisons with other regularization techniques. Here it is seen that the first-order method gives smoother solutions than the zero-order method with approximately the same reconstruction error in the supremum-norm (see Figure 5.2). It is also seen (Figure 5.3) that our method is less sensitive to over-regularization than the zero-order 
method. Moreover, the numerical effects of the violation of the stability condition $\left(\mathrm{A}_{3}\right)$ is considered.

2. A first-order sequential regularization method. We consider the linear Volterra equation of the first kind

$$
V u=f,
$$

where

$$
V u(t)=\int_{0}^{t} k(t-s) u(s) d s \quad \text { for } t \in[0,1]
$$

with $k, f \in \mathcal{C}[0,1]$. It follows from the Arzelà-Ascoli theorem that the integral operator $(2.2)$ is compact on $\mathcal{C}[0,1]$. Hence, a solution to $(2.1)$, if it exists, does not depend continuously on the right-hand side $f$. In the following, we define a family of approximating well-posed problems which allow us to solve (2.1) in a numerically stable way.

Let us develop the main idea for a discretized version of (2.1). Let $0=t_{0}<$ $t_{1}<\cdots<t_{N}=1$ with $t_{i}=\frac{i}{N}$ for $i=0, \ldots, N$ be a uniform partition of the interval $[0,1]$. We set $h=\frac{1}{N}$. For $0 \leq i \leq N$ we define the piecewise affine basis functions $\varphi_{i}^{N}$ by $\varphi_{i}^{N} \in \mathcal{C}[0,1]$, where $\varphi_{i}^{N}$ is affine on $\left[t_{j-1}, t_{j}\right]$ for $1 \leq j \leq N$ and $\varphi_{i}^{N}\left(t_{j}\right)=\delta_{i, j}$ for $0 \leq j \leq N$. Let $\mathcal{B}_{N} \subset \mathcal{C}[0,1]$ be the space of continuous, piecewise affine functions with respect to the partition $\left\{t_{i}\right\}_{i=0}^{N}$ :

$$
\begin{aligned}
\mathcal{B}_{N}=\left\{\varphi \in \mathcal{C}[0,1]:\left.\varphi\right|_{\left[t_{i-1}, t_{i}\right]}\right. & \left.=a_{i} t+b_{i} ; a_{i}, b_{i} \in \mathbb{R} \text { for } i=1, \ldots, N\right\} \\
& =\operatorname{span}\left\{\varphi_{i}^{N}: 0 \leq i \leq N\right\} .
\end{aligned}
$$

It is helpful to introduce the restriction operator $r_{N}: \mathcal{C}[0,1] \rightarrow \mathbb{R}^{N+1}$ by

$$
\left[r_{N}(u)\right]_{i}=u\left(t_{i}\right) \quad \text { for } 0 \leq i \leq N .
$$

In the collocation method an approximation $u^{N} \in \mathcal{B}_{N}$ to the solution $u$ is obtained by solving the system of equations

$$
r_{N} V u^{N}=r_{N} f
$$

We set $\mathbf{u}^{N}=\left(u_{0}^{N}, \ldots, u_{N}^{N}\right)^{t}=r_{N} u^{N}$ and $\mathbf{f}^{N}=\left(f_{0}^{N}, \ldots, f_{N}^{N}\right)^{t}=r_{N} f$. It is obvious from (2.1) that $u_{0}^{N}$ can be chosen arbitrarily and $f_{0}^{N}=0$ has to be satisfied. For $i \geq 1$ the collocation assumption yields the product integration-type relation

$$
\int_{0}^{t_{i}} k\left(t_{i}-s\right)\left(\sum_{j=0}^{i} u_{j}^{N} \varphi_{j}^{N}(s)\right) d s=f_{i}^{N} .
$$

Equation (2.4) is a linear system for the unknown vector $\mathbf{u}^{N}$. We shall write the system (2.4) in matrix form:

$$
A^{N} \mathbf{u}^{N}=\mathbf{f}^{N}
$$

We define

$$
\Delta_{i, j}=\int_{0}^{t_{i}} k\left(t_{i}-s\right) \varphi_{j}^{N}(s) d s
$$


for $0 \leq i, j \leq N$. Obviously, $\Delta_{0,0}=0$. Using the change of variables $\tau=t_{i}-s$, in (2.6) it is easily seen that $\Delta_{i, j}$ depends only on $m=i-j$ for $i, j \geq 1$. Thus, we can define

$$
\Delta_{m}=\Delta_{m, 1} \quad \text { for } m \geq 1 \text {, }
$$

and we find $\Delta_{m}=\Delta_{m+i-1, i}$ for $1 \leq m \leq N$ and $1 \leq i \leq N-m+1$. With this equation, (2.4), and (2.6) we obtain the system matrix

$$
A^{N}=\left(\begin{array}{cc}
0 & 0 \\
\mathbf{d} & \tilde{A}^{N}
\end{array}\right)
$$

where $\mathbf{d}=\left(\Delta_{1,0}, \ldots, \Delta_{N, 0}\right)^{t}$ and $\tilde{A}^{N}$ is given by the semicirculant matrix

$$
\tilde{A}^{N}=\left(\begin{array}{ccccc}
\Delta_{1} & & & & \\
\Delta_{2} & \Delta_{1} & & 0 & \\
\Delta_{3} & \Delta_{2} & \Delta_{1} & & \\
\vdots & & \ddots & \ddots & \\
\Delta_{N} & & \cdots & \Delta_{2} & \Delta_{1}
\end{array}\right) .
$$

Obviously, $A^{N}$ is singular. We have already seen that we have free choice for $u_{0}^{N}$. But once we have chosen $u_{0}^{N},(2.5)$ can be solved sequentially for the remaining variables $u_{1}^{N}, \ldots, u_{N}^{N}$, i.e., we have

$$
u_{i}^{N}=\frac{1}{\Delta_{1}}\left(f_{i}^{N}-\Delta_{i, 0} u_{0}^{N}-\sum_{j=1}^{i-1} \Delta_{i-j+1} u_{j}^{N}\right) .
$$

If we replace the right-hand side in (2.8) by a perturbation $f_{i}^{N}+\delta f_{i}^{N}$ we obtain

$$
\delta u_{i}^{N}=\frac{\delta f_{i}^{N}}{\Delta_{1}}
$$

for the error in the solution $u_{i}^{N}$. Thus, noise in the data is amplified by a factor $\frac{1}{\Delta_{1}}$ in the solution. The condition of the linear system (2.5), therefore, depends crucially on the magnitude of

$$
\Delta_{1}=\frac{1}{h} \int_{0}^{h} k(s)(h-s) d s
$$

We have

$$
\Delta_{1} \approx k(0) \frac{h}{2 !}+k^{\prime}(0) \frac{h^{2}}{3 !}+k^{\prime \prime}(0) \frac{h^{3}}{4 !}+\cdots
$$

Thus, for small $h$, the magnitude of $\Delta_{1}$ is determined by the first nonvanishing derivative of $k$ at $t=0$. This gives rise to the following definition. We say that the kernel $k$ is $\nu$-smoothing if

$$
k \in \mathcal{C}^{(\nu)}[0,1] \text { and } k^{(l)}(0)=0 \text { for } l=0, \ldots, \nu-2 \text {, and } k^{(\nu-1)}(0) \neq 0,
$$

where $k^{(l)}$ denotes the $l$ th derivative of $k$. We see that (2.5) becomes more and more ill-conditioned the larger $\nu$ is. We assume for the rest of the paper that $k$ is $\nu$-smoothing with $\nu \geq 1$. 
We pursue the following idea to find an approximating solution to (2.5) which is stable and sequential. We do not try to match every data point $f_{i}^{N}$ exactly. Instead, we choose the solution $u^{N}$ to be an affine function on the interval $\left[t_{i}, t_{i+1}\right]$, for which an (affine) continuation onto the interval $\left[t_{i}, t_{i+r+1}\right]$ fits the data $\left(f_{i+1}^{N}, \ldots, f_{i+r+1}^{N}\right)$ in a weighted least-squares sense, for some $r>0$. Thus, we also take future information into account to calculate the solution on the current interval. Let us make this idea precise. We consider $u^{N}=\sum_{i=0}^{N} u_{i}^{N} \varphi_{i}^{N} \in \mathcal{B}^{N}$, and we set

$$
\left(u_{i}^{N}\right)^{\prime}=\frac{1}{h}\left(u_{i+1}^{N}-u_{i}^{N}\right)
$$

for $0 \leq i \leq N-1$. Moreover, we define the operators $q_{i, N}: \mathcal{B}^{N} \rightarrow \mathcal{B}^{N}$ with $0 \leq i \leq$ $N-1$ by

$$
q_{i, N} u^{N}(t)= \begin{cases}u^{N}(t) & \text { if } t<t_{i}, \\ u_{i}^{N}+\left(u_{i}^{N}\right)^{\prime}\left(t-t_{i}\right) & \text { if } t \geq t_{i} .\end{cases}
$$

Assuming that $u_{j}^{N}$ is already determined for all $j \leq i$, we determine $u_{i+1}^{N}$ by minimizing

$$
\sum_{l=0}^{r} \omega_{l}\left|\left[r_{N} V\left(q_{i, N} u^{N}\right)\right]_{i+l+1}-f_{i+l+1}^{N}\right|^{2}
$$

Here $r \geq 0$ is the number of future time-steps which are considered. For $r=0$, we obtain the collocation scheme $(2.3)$. The numbers $\omega_{0}, \ldots, \omega_{r}$ are given positive weights. The significance of the weights $\omega_{l}$ will become apparent in section 3 , where we shall introduce the stability conditions $\left(A_{1}\right)-\left(A_{3}\right)$. Using a nonuniform weight will give us more flexibility to satisfy the mentioned conditions. See also the discussion at the end of section 5 .

In general, $(2.11)$ is solved for $\left(u_{i}^{N}\right)^{\prime}$ with known $\left(u_{0}^{N}, \ldots, u_{i}^{N}\right)$. Setting $u_{i+1}^{N}=$ $u_{i}^{N}+h\left(u_{i}^{N}\right)^{\prime}$, we can proceed to the next step. Only in the first step, $i=0$, we have to solve for two unknowns $u_{0}^{N}$ and $\left(u_{0}^{N}\right)^{\prime}$.

We define

$$
\begin{aligned}
\kappa_{l} & =\int_{0}^{t_{l+1}} k(s) d s, \\
\lambda_{l} & =\int_{0}^{t_{l+1}}\left(t_{l+1}-s\right) k(s) d s
\end{aligned}
$$

for $l=0, \ldots, r$. From (2.10), using a transformation of the domain of integration we obtain

$$
\left[r_{N} V\left(q_{i, N} u^{N}\right)\right]_{i+l+1}=\int_{0}^{t_{i}} k\left(t_{i+l+1}-s\right) u^{N}(s) d s+\kappa_{l} u_{i}^{N}+\lambda_{l}\left(u_{i}^{N}\right)^{\prime},
$$

where $0 \leq i$ and $0 \leq l \leq r$. In the case $i=0$ the necessary optimality conditions with respect to the variables $u_{0}^{N}$ and $\left(u_{0}^{N}\right)^{\prime}$ for problem (2.11) are, therefore, given by

$$
\begin{aligned}
& \sum_{l=0}^{r} \omega_{l} \kappa_{l}\left(\kappa_{l} u_{0}^{N}+\lambda_{l}\left(u_{0}^{N}\right)^{\prime}\right)=\sum_{l=0}^{r} \omega_{l} \kappa_{l} f_{l+1}^{N}, \\
& \sum_{l=0}^{r} \omega_{l} \lambda_{l}\left(\kappa_{l} u_{0}^{N}+\lambda_{l}\left(u_{0}^{N}\right)^{\prime}\right)=\sum_{l=0}^{r} \omega_{l} \lambda_{l} f_{l+1}^{N} .
\end{aligned}
$$


For $i \geq 1$, the optimality condition for (2.11) with respect or the single variable $\left(u_{i}^{N}\right)^{\prime}$ is given by

$$
\sum_{l=0}^{r} \omega_{l} \lambda_{l}\left(\int_{0}^{t_{i}} k\left(t_{i+l+1}-s\right) u^{N}(s) d s+\kappa_{l} u_{i}^{N}+\lambda_{l}\left(u_{i}^{N}\right)^{\prime}\right)=\sum_{l=0}^{r} \omega_{l} \lambda_{l} f_{i+l+1}^{N} .
$$

We can write (2.12), (2.13), and (2.14) in the following more compact form:

$$
\begin{gathered}
\left(\begin{array}{ll}
\alpha & \beta \\
\beta & \gamma
\end{array}\right)\left(\begin{array}{l}
u_{0} \\
u_{0}^{\prime}
\end{array}\right)=\left(\begin{array}{c}
\hat{g} \\
\hat{f}_{0}
\end{array}\right), \\
\sum_{j=0}^{i} \tilde{\Delta}_{i, j} u_{j}+\beta u_{i}+\gamma u_{i}^{\prime}=\hat{f}_{i} \quad \text { for every } i \geq 1, \\
u_{i+1}=u_{i}+h u_{i}^{\prime} \quad \text { for every } i \geq 0 .
\end{gathered}
$$

Here we omitted the discretization index $N$ and we set

$$
\begin{aligned}
& \alpha=\sum_{l=0}^{r} \omega_{l} \kappa_{l}^{2}, \quad \beta=\sum_{l=0}^{r} \omega_{l} \kappa_{l} \lambda_{l}, \quad \gamma=\sum_{l=0}^{r} \omega_{l} \lambda_{l}^{2}, \\
& \hat{g}=\sum_{l=0}^{r} \omega_{l} f_{l+1} \kappa_{l}, \quad \hat{f}_{i}=\sum_{l=0}^{r} \omega_{l} f_{i+l+1} \lambda_{l} \text { for } i \geq 0,
\end{aligned}
$$

and

$$
\tilde{\Delta}_{i, j}=\sum_{l=0}^{r} \omega_{l} \lambda_{l} \int_{0}^{t_{i}} k\left(t_{i+l+1}-s\right) \varphi_{j}(s) d s \text { for } 0 \leq j \leq i .
$$

If we solve the $2 \times 2$-system (2.15a) for the initial values $u_{0}$ and $u_{0}^{\prime}$, and if we assign $u_{1}=u_{0}+h u_{0}^{\prime}$, then we can solve (2.15b) sequentially by forward substitution:

$$
\begin{aligned}
u_{i}^{\prime} & =\frac{1}{\gamma}\left(\hat{f}_{i}-\beta u_{i}-\sum_{j=0}^{i} \tilde{\Delta}_{i, j} u_{j}\right), \\
u_{i+1} & =u_{i}+h u_{i}^{\prime} \text { for } i \geq 1 .
\end{aligned}
$$

The system (2.15) can be interpreted as a collocation scheme for a second-kind Volterra integro-differential equation. This is seen as follows. Suppose that $\omega$ is a positive, regular Borel measure on $[0, \rho]$ for $\rho>0$. We set

$$
\begin{aligned}
\kappa(\tau) & =\int_{0}^{\tau} k(s) d s \\
\lambda(\tau) & =\int_{0}^{\tau}(\tau-s) k(s) d s \\
a & =\int_{0}^{\rho} \kappa^{2}(\tau) d \omega(\tau), \quad b=\int_{0}^{\rho} \kappa(\tau) \lambda(\tau) d \omega(\tau), \quad c=\int_{0}^{\rho} \lambda^{2}(\tau) d \omega(\tau), \\
\tilde{g} & =\int_{0}^{\rho} f(\tau) \kappa(\tau) d \omega(\tau) \\
\tilde{f}(t) & =\int_{0}^{\rho} \lambda(\tau) f(t+\tau) d \omega(\tau) \\
\tilde{k}(t) & =\int_{0}^{\rho} \lambda(\tau) k(t+\tau) d \omega(\tau) .
\end{aligned}
$$


Note that the above defined expressions (2.18)-(2.21) depend on $\rho$, both directly, since $\rho$ appears in the upper bound of the integrals, and indirectly via $\omega=\omega(\rho)$. Obviously, we have $\kappa\left(t_{l+1}\right)=\kappa_{l}$, and $\lambda\left(t_{l+1}\right)=\lambda_{l}$, for $l=0, \ldots, r$. Let $\delta_{x}(\tau)$ denote the Dirac point measure located at $\tau=x$ and let $\rho=(r+1) h$. Then, for the special case where $\omega$ is the sum of discrete point measures given by

$$
\omega=\sum_{l=0}^{r} \omega_{l} \delta_{t_{l+1}}(\tau)
$$

we have $a=\alpha, b=\beta, c=\gamma, \tilde{g}=\hat{g}$, and $\tilde{f}\left(t_{i}\right)=\hat{f}_{i}$ for $i=0,1,2, \ldots$ Equation $(2.15 \mathrm{~b})$ can then be written as

$$
r_{N} \tilde{V} u+b u+c u^{\prime}=r_{N} \tilde{f}
$$

with

$$
\tilde{V} u(t)=\int_{0}^{t} \tilde{k}(t-s) u(s) d s .
$$

Thus, (2.15b) is a collocation discretization of the Volterra integro-differential equation

$$
\begin{aligned}
& \left(\begin{array}{ll}
a & b \\
b & c
\end{array}\right)\left(\begin{array}{c}
u(0) \\
u^{\prime}(0)
\end{array}\right)=\left(\begin{array}{c}
\tilde{g} \\
\tilde{f}(0)
\end{array}\right) \\
& \int_{0}^{t} \tilde{k}(t-s) u(s) d s+b u(t)+c u^{\prime}(t)=\tilde{f}(t)
\end{aligned}
$$

for the case that $\omega$ is given by (2.22), $u=u^{N} \in \mathcal{B}_{N}$, and $u_{i}^{\prime}$ is interpreted as rightsided derivative at $t_{i}$. Note that the second component in (2.23a) is actually $(2.23 \mathrm{~b})$ at time $t=0$. We prefer, however, the redundant notation (2.23a) since it highlights the role of the initial values $\left(u(0), u^{\prime}(0)\right)$.

The following section is devoted to the analysis of the Volterra integro-differential equation (2.23).

3. Stability estimates. We set

$$
k_{\nu}=k^{(\nu-1)}(0) \neq 0 .
$$

(Recall our assumption that $k$ is $\nu$-smoothing.) We assume in the following without loss of generality that $k_{\nu}>0$. Note that this assumption implies that $k(s)>0$ for $s$ small enough. Therefore, we usually omit absolute value bars in estimations if we integrate $k$ over small intervals of the form $[0, \rho]$.

For every $\rho \in(0, R]$ let $\omega_{\rho}$ be a positive, regular Borel measure on $[0, \rho]$. If we do not want to stress the dependence of $\omega_{\rho}$ on $\rho$, we frequently omit the subscript. Here and in the following we use standard Landau $o$-symbols to denote functions vanishing at zero. Throughout the rest of the paper we use the following assumptions on the moments of the measures $\omega_{\rho}$.

There exists an integer $s \geq 0$ and there exist positive numbers $C_{j}$ independent of $\rho$ such that

$$
\int_{0}^{\rho} \tau^{j} d \omega_{\rho}(\tau)=\rho^{s+j}\left(C_{j}+o(\rho)\right) \text { for } j=0, \ldots, 2 \nu+2
$$


with

$$
C_{2 \nu} C_{2 \nu+2}-C_{2 \nu+1}^{2}>0 \text {. }
$$

$$
\text { All roots of the polynomial } \sum_{l=0}^{\nu+1} \frac{C_{\nu+l+1}}{l !} x^{l} \text { have negative real parts. }
$$

Remark 1. By assumption $\left(\mathrm{A}_{1}\right)$ we have

$$
\left(\int_{0}^{\rho} \tau^{2 \nu+1} d \omega\right)^{2}=\rho^{2 s+4 \nu+2}\left(C_{2 \nu+1}^{2}+o(\rho)\right)
$$

On the other hand, we have

$$
\begin{aligned}
\left(\int_{0}^{\rho} \tau^{2 \nu+1} d \omega\right)^{2} & =\left(\int_{0}^{\rho} \tau^{\nu} \tau^{\nu+1} d \omega\right)^{2} \leq \int_{0}^{\rho} \tau^{2 \nu} d \omega \int_{0}^{\rho} \tau^{2 \nu+2} d \omega \\
& =\rho^{2 s+4 \nu+2}\left(C_{2 \nu} C_{2 \nu+2}+o(\rho)\right) .
\end{aligned}
$$

Hence, we find that $C_{2 \nu} C_{2 \nu+2}-C_{2 \nu+1}^{2} \geq 0$ is always satisfied. Condition $\left(\mathrm{A}_{2}\right)$ is, therefore, only the requirement that the inequality be strict.

Remark 2. The role of condition $\left(\mathrm{A}_{3}\right)$ will become apparent throughout the subsequent considerations. It is a stability criterion for (2.23).

Before we investigate solvability and well-posedness of the integro-differential equation (2.23) we give some asymptotic estimates which we derive from $\left(\mathrm{A}_{1}\right)$ and the definitions of the respective terms in section 1 . We have

$$
\begin{aligned}
k^{(l)}(s) & =s^{\nu-l-1}\left(\frac{k_{\nu}}{(\nu-l-1) !}+o(s)\right) \quad \text { for } l=0, \ldots, \nu-1, \\
\kappa(\tau) & =\tau^{\nu}\left(\frac{k_{\nu}}{\nu !}+o(\tau)\right) \\
\lambda(\tau) & =\tau^{\nu+1}\left(\frac{k_{\nu}}{(\nu+1) !}+o(\tau)\right) \\
\int_{0}^{\rho} \kappa(\tau) d \omega(\tau) & =\rho^{s+\nu}\left(C_{\nu} \frac{k_{\nu}}{\nu !}+o(\rho)\right) \\
\int_{0}^{\rho} \lambda(\tau) d \omega(\tau) & =\rho^{s+\nu+1}\left(C_{\nu+1} \frac{k_{\nu}}{(\nu+1) !}+o(\rho)\right) \\
a & =\rho^{s+2 \nu}\left(C_{2 \nu} \frac{k_{\nu}^{2}}{(\nu !)^{2}}+o(\rho)\right) \\
b & =\rho^{s+2 \nu+1}\left(C_{2 \nu+1} \frac{k_{\nu}^{2}}{\nu !(\nu+1) !}+o(\rho)\right) \\
c & =\rho^{s+2 \nu+2}\left(C_{2 \nu+2} \frac{k_{\nu}^{2}}{((\nu+1) !)^{2}}+o(\rho)\right)
\end{aligned}
$$

From definition (2.21) it follows easily that $\tilde{k} \in \mathcal{C}^{\nu}[0,1]$. Using (3.1), (3.3), and $\left(\mathrm{A}_{1}\right)$, we obtain

$$
\tilde{k}^{(l)}(0)=\rho^{s+2 \nu-l}\left(C_{2 \nu-l} \frac{k_{\nu}^{2}}{(\nu+1) !(\nu-l-1) !}+o(\rho)\right) \quad \text { for } l=0, \ldots, \nu-1 .
$$


Remark 3. We can use the leading term in (3.5)

$$
\hat{q}=\rho^{s+\nu+1} C_{\nu+1} \frac{k_{\nu}}{(\nu+1) !}
$$

as a normalizing factor for (2.23b). Multiplying (2.23b) by $\hat{q}^{-1}$ leads to an equation of the form

$$
\hat{V} u(t)=\int_{0}^{t} \hat{k}(t-\tau) u(\tau) d \tau+\hat{b} u(t)+\hat{c} u^{\prime}(t)=\hat{f}(t) .
$$

Using $\left(\mathrm{A}_{1}\right)$ and the asymptotic expressions (3.4)-(3.8), it can be shown that $\hat{f}(t) \rightarrow$ $f(t), \int_{0}^{t} \hat{k}(t-\tau) u(\tau) d \tau \rightarrow \int_{0}^{t} k(t-\tau) u(\tau) d \tau$ uniformly on $[0,1], \hat{b} \rightarrow 0$, and $\hat{c} \rightarrow 0$ as $\rho \rightarrow 0$. Thus, in the limit $\rho \rightarrow 0$, the normalized equation (3.10) converges to the original equation (1.1) uniformly on $[0,1]$.

For the purpose of analysis we prefer to work with the integral equation $(2.23 \mathrm{~b})$ instead of (3.10), since dividing by $\hat{q}$ makes many expressions even more cumbersome to calculate and estimate. From the numerical point of view the normalization (3.10) is advisable.

We now prove solvability and well-posedness for (2.23) for a general continuous right-hand side $f$.

Proposition 3.1. Let $f$ be continuous on $[0,1+R)$ for some $R>0$, and let $k$ be $\nu$-smoothing with $\nu>0$. Let $a, b$, and $c$ be given by (2.18) and suppose that $\tilde{g}$, $\tilde{f}$ and $\tilde{k}$ are defined by (2.19), (2.20), and (2.21), respectively. Assume, moreover, that assumptions $\left(A_{1}\right)$ and $\left(A_{2}\right)$ hold. Then there exists a $\rho_{0}>0$ such that problem (2.23) has a unique solution $u \in \mathcal{C}^{1}[0,1]$ for all $\rho \leq \rho_{0}$. Moreover, there exist constants $\mathcal{M}, \overline{\mathcal{M}}>0$ independent of $\rho$ such that

$$
\|u\|_{\mathcal{C}[0,1]} \leq \frac{\mathcal{M}}{\rho^{\nu+1}} \exp \left(\frac{\mathcal{M}}{\rho^{\nu+1}}\right)\|f\|_{\mathcal{C}[0,1+\rho]}
$$

and

$$
\left\|u^{\prime}\right\|_{\mathcal{C}[0,1]} \leq \frac{\overline{\mathcal{M}}}{\rho^{2 \nu+2}} \exp \left(\frac{\mathcal{M}}{\rho^{\nu+1}}\right)\|f\|_{\mathcal{C}[0,1+\rho]}
$$

Proof. It is easily seen from the definition (2.20) that $\tilde{f}$ is continuous on $[0,1+R-$ $\rho$ ), and by (3.7) and (3.8) that $b \neq 0$ and $c \neq 0$ for $\rho$ small enough. Hence, it follows by standard Picard iteration arguments (see Burton [2, pp. 23-24]) that (2.23b) has a unique solution $u \in \mathcal{C}^{1}[0,1]$ provided that we can solve the linear system $(2.23 \mathrm{a})$ uniquely for $u(0)$. Using (3.6) and (3.8) we find

$$
\operatorname{det}\left(\begin{array}{ll}
a & b \\
b & c
\end{array}\right)=\rho^{2 s+4 \nu+2} \frac{k_{\nu}^{4}}{(\nu !)^{2}((\nu+1) !)^{2}}\left(C_{2 \nu} C_{2 \nu+2}-C_{2 \nu+1}^{2}+o(\rho)\right)>0
$$

for $\rho$ small enough due to assumption $\left(\mathrm{A}_{2}\right)$. Thus, the matrix in (2.23a) is invertible, and solvability for $(2.23 \mathrm{~b})$ follows.

Note that the mappings $f \mapsto(\tilde{g}, \tilde{f}(0)),(\tilde{g}, \tilde{f}(0)) \mapsto u(0)$, and $f \mapsto \tilde{f}$ are all linear. Therefore, the solution operator $f \mapsto u(f)$, where $u(f)$ is the solution to (2.23), is linear. 
Now we estimate $\tilde{g}$ and $\tilde{f}(0)$ in terms of $\|f\|_{\mathcal{C}[0,1+\rho]}$. With (2.19), (2.20), (3.4), and (3.5) we have

$$
\tilde{g} \leq \rho^{s+\nu}\left(C_{\nu} \frac{k_{\nu}}{\nu !}+o(\rho)\right)\|f\|_{\mathcal{C}[0,1+\rho]}
$$

and

$$
\tilde{f(0)} \leq \rho^{s+\nu+1}\left(C_{\nu+1} \frac{k_{\nu}}{(\nu+1) !}+o(\rho)\right)\|f\|_{\mathcal{C}[0,1+\rho]} .
$$

Thus, using (3.6), (3.7), and (3.8) we find

$$
\begin{aligned}
|u(0)| & =\left|\frac{c \tilde{g}-b \tilde{f}(0)}{a c-b^{2}}\right| \\
& \leq \frac{1}{\rho^{\nu}}\left|\frac{\nu !}{k_{\nu}} \frac{C_{2 \nu+2} C_{\nu}+C_{2 \nu+1} C_{\nu+1}}{C_{2 \nu+2} C_{2 \nu}-C_{2 \nu+1}^{2}}+o(\rho)\right|\|f\|_{\mathcal{C}[0,1+\rho]} \\
& \leq \frac{1}{\rho^{\nu}} m_{1}\|f\|_{\mathcal{C}[0,1+\rho]}
\end{aligned}
$$

for some constant $m_{1}>0$ independent of $\rho$. With (3.8) we obtain

$$
|c u(0)| \leq \rho^{s+\nu+2} m_{2}\|f\|_{\mathcal{C}[0,1+\rho]}
$$

for some constant $m_{2}>0$ independent of $\rho$.

From (3.5) and definition (2.20) we derive the following estimate:

$$
\begin{aligned}
\left|\int_{0}^{t} \tilde{f}(s) d s\right| & \leq \rho^{s+\nu+1}\left(C_{\nu+1} \frac{k_{\nu}}{(\nu+1) !}+o(\rho)\right)\|f\|_{\mathcal{C}[0,1+\rho]} \\
& \leq \rho^{s+\nu+1} m_{3}\|f\|_{\mathcal{C}[0,1+\rho]}
\end{aligned}
$$

for all $t \in[0,1]$ with some constant $m_{3}>0$ independent of $\rho$. Estimates (3.15) and (3.16) yield that there exists a constant $m_{4}>0$ independent of $\rho$ such that

$$
\left|\int_{0}^{t} \tilde{f}(s) d s+c u(0)\right| \leq \rho^{s+\nu+1} m_{4}\|f\|_{\mathcal{C}[0,1+\rho]}
$$

for all $t \in[0,1]$.

We set

$$
K(t)=\int_{0}^{t} \tilde{k}(s) d s+b
$$

Using (3.5) and (3.7) we get

$$
\begin{aligned}
|K(t)| & \leq \rho^{s+\nu+1}\left(C_{\nu+1} \frac{k_{\nu}}{(\nu+1) !}+o(\rho)\right)\|k\|_{\mathcal{C}[0,1+\rho]}+b \\
& \leq \rho^{s+\nu+1} m_{5}
\end{aligned}
$$

for all $t \in[0,1]$ with some constant $m_{5}$ depending on $k$ but not on $\rho$. Moreover, it is obvious from (3.8) that there exists a constant $m_{6}>0$ independent of $\rho$ such that

$$
\frac{1}{c} \leq \frac{m_{6}}{\rho^{s+2 \nu+2}}
$$


for all $\rho$ sufficiently small. We set

$$
\mathcal{M}=\max \left(m_{4} m_{6}, m_{5} m_{6}\right) .
$$

Integrating $(2.23 \mathrm{~b})$ with respect to $t$ yields

$$
\int_{0}^{t} K(t-s) u(s) d s+c u(t)=\int_{0}^{t} \tilde{f}(s) d s+c u(0) .
$$

Hence, by (3.17), (3.18), (3.19), and (3.20) we obtain

$$
|u(t)| \leq \frac{\mathcal{M}}{\rho^{\nu+1}}\left(\|f\|_{\mathcal{C}[0,1+\rho]}+\int_{0}^{t}|u(s)| d s\right)
$$

for all $t \in[0,1]$. Then Gronwall's inequality implies

$$
|u(t)| \leq \frac{\mathcal{M}}{\rho^{\nu+1}} \exp \left(\frac{\mathcal{M} t}{\rho^{\nu+1}}\right)\|f\|_{\mathcal{C}[0,1+\rho]} .
$$

Thus, (3.11) follows. From (2.23b) we conclude

$$
\left|u^{\prime}(t)\right| \leq \frac{1}{c}\left(\left(1+\|\tilde{k}\|_{\mathcal{C}[0,1]}\right)\|u\|_{\mathcal{C}[0,1]}+\|\tilde{f}\|_{\mathcal{C}[0,1]}\right)
$$

for all $t \in[0,1]$. With (3.21), (3.7), (3.8), and (3.5) we find that there exists a constant $\overline{\mathcal{M}}>0$ such that

$$
\left|u^{\prime}(t)\right| \leq \frac{\overline{\mathcal{M}}}{\rho^{2 \nu+2}} \exp \left(\frac{\mathcal{M} t}{\rho^{\nu+1}}\right)\|f\|_{\mathcal{C}[0,1+\rho]}
$$

for all $t \in[0,1]$. This completes the proof.

The study of the homogeneous equation

$$
u^{\prime}(t)=-\frac{b}{c} u(t)-\frac{1}{c} \int_{0}^{t} \tilde{k}(t-s) u(s) d s \quad \text { for } t \in[0,1]
$$

will help us to obtain estimates for different corresponding inhomogeneous problems. We use the transform $y(t)=u(\rho t)$ to get the integro-differential equation

$$
y^{\prime}(t)=-\frac{b \rho}{c} y(t)-\frac{\rho^{2}}{c} \int_{0}^{t} \tilde{k}(\rho(t-s)) y(s) d s \quad \text { for } t \in\left[0, \frac{1}{\rho}\right] .
$$

Let $z_{\rho}$ denote the fundamental solution to (3.24), i.e., the solution to (3.24) with $z_{\rho}(0)=1$. The following estimates for $z_{\rho}$ will be useful in combination with the variation of constants formula.

Lemma 3.2. Assume that conditions $\left(\mathrm{A}_{1}\right),\left(\mathrm{A}_{2}\right)$, and $\left(\mathrm{A}_{3}\right)$ hold. Then there exist constants $\tilde{C}>0$ (depending on $\omega$ ) and $m>0$ (depending on $k$ and $\omega$ ) such that

$$
\left|z_{\rho}(t)\right| \leq m \quad \text { for all } t \in\left[0, \frac{1}{\rho}\right]
$$

and

$$
\left\|z_{\rho}\right\|_{L^{1}\left(0, \frac{1}{\rho}\right)} \leq m
$$


independently of $\rho$ if

$$
\frac{\left\|k^{(\nu)}\right\|_{L^{1}(0,1)}}{k_{\nu}}<\tilde{C}
$$

Proof. Since $\tilde{k}$ is $\nu$ times continuously differentiable we can differentiate (3.24) with respect to $t$. Setting $y=z_{\rho}$ we obtain

$$
\begin{aligned}
z_{\rho}^{(j+1)}(t)=-\frac{b \rho}{c} z_{\rho}^{(j)}(t) & -\sum_{l=0}^{j-1} \frac{\tilde{k}^{(l)}(0) \rho^{l+2}}{c} z_{\rho}^{(j-l-1)}(t) \\
& -\frac{\rho^{j+2}}{c} \int_{0}^{t} \tilde{k}^{(j)}(\rho(t-s)) z_{\rho}(s) d s
\end{aligned}
$$

for $j=0, \ldots, \nu$. We set

$$
\alpha_{l}=\alpha_{l}(\nu)=\frac{C_{\nu+l+1}}{C_{2 \nu+2}} \frac{(\nu+1) !}{l !} \text { for } l=0, \ldots, \nu .
$$

Using (3.7), (3.8), and (3.9) we find for $j=\nu$ that

$$
z_{\rho}^{(\nu+1)}(t)=-\sum_{l=0}^{\nu}\left(\alpha_{\nu-l}+o(\rho)\right) z_{\rho}^{(\nu-l)}(t)-\frac{\rho^{\nu+2}}{c} \int_{0}^{t} \tilde{k}^{(\nu)}(\rho(t-s)) z_{\rho}(s) d s .
$$

We write the $(\nu+1)$-order equation (3.30) as a system of integro-differential equations in the usual way, setting

$$
\mathbf{z}_{\rho}=\left(z_{\rho}^{(0)}, \ldots, z_{\rho}^{(\nu)}\right)^{T} .
$$

With this we obtain the system

$$
\mathbf{z}_{\rho}^{\prime}(t)=A \mathbf{z}_{\rho}(t)+M_{\rho} \mathbf{z}_{\rho}(t)+\int_{0}^{t} D_{\rho}(t-s) \mathbf{z}_{\rho}(s) d s,
$$

where

$$
A=\left(\begin{array}{cccc}
0 & 1 & & 0 \\
\vdots & \ddots & \ddots & \\
0 & \ldots & 0 & 1 \\
-\alpha_{0} & -\alpha_{1} & \ldots & -\alpha_{\nu}
\end{array}\right)
$$

$$
\left\|M_{\rho}\right\| \rightarrow 0 \text { as } \rho \rightarrow 0
$$

and

$$
D_{\rho}(t)=\left(\begin{array}{cccc}
0 & \ldots & & 0 \\
\vdots & & & \\
0 & & & \vdots \\
-\frac{\rho^{\nu+2}}{c} \tilde{k}^{(\nu)}(\rho t) & 0 & \ldots & 0
\end{array}\right)
$$


Now we consider the relation between the initial conditions $z_{\rho}(0)=1$ and $\mathbf{z}_{\rho}(0)=$ $\left(z_{\rho}^{(0)}(0), \ldots, z_{\rho}^{(\nu)}(0)\right)^{T}$. Using (3.28) we find

$$
z_{\rho}^{(j+1)}(0)=-\sum_{l=0}^{j}\left(\alpha_{\nu-l}+o(\rho)\right) z_{\rho}^{(j-l)}(0) \quad \text { for } j=0, \ldots, \nu .
$$

It is seen by induction that there exists a constant $d>0$ independent of $\rho$ such that

$$
\left|\mathbf{z}_{\rho}(0)\right| \leq d
$$

for all $\rho$ sufficiently small.

Now we prove the estimates for $\left|z_{\rho}(t)\right|$ and $\left\|z_{\rho}\right\|_{L^{1}\left(0, \frac{1}{\rho}\right)}$. The eigenvalues of the matrix $A$ are given by the roots of the polynomial

$$
q(x)=(-1)^{\nu+1}\left(\sum_{l=0}^{\nu} \alpha_{l} x^{l}+x^{\nu+1}\right) .
$$

Upon multiplying $q$ by $\frac{C_{2 \nu+2}}{(\nu+1) !}$ we see that the roots of $q$ coincide with the roots of the polynomial

$$
\sum_{l=0}^{\nu+1} \frac{C_{\nu+l+1}}{l !} x^{l} .
$$

By assumption $\left(\mathrm{A}_{3}\right)$, all roots of the polynomial (3.36) have negative real parts. Then, for sufficiently small $\rho$, all real parts of eigenvalues of $A_{\rho}=A+M_{\rho}$ are negative and bounded away from zero. With the notation $A_{0}=A$ we define

$$
B_{\rho}=\int_{0}^{\infty}\left(\exp \left(A_{\rho} t\right)\right)^{T} \exp \left(A_{\rho} t\right) d t
$$

for $\rho \geq 0$ sufficiently small. The matrix $B_{\rho}$ is well defined, symmetric, positive definite, and satisfies

$$
A_{\rho}^{T} B_{\rho}+B_{\rho} A_{\rho}=-I
$$

(cf. [2, p. 124]). Using Lebesgue's dominated convergence theorem we find $B_{\rho} \rightarrow B_{0}$ as $\rho \rightarrow 0$. From this it follows that there exist positive constants $L, K$, and $R$ such that

$$
\begin{aligned}
|\mathbf{x}| & \geq 2 L\left(\mathbf{x}^{T} B_{\rho} \mathbf{x}\right)^{\frac{1}{2}}, \\
\left|B_{\rho} \mathbf{x}\right| & \leq K\left(\mathbf{x}^{T} B_{\rho} \mathbf{x}\right)^{\frac{1}{2}}, \\
|\mathbf{x}| & \leq R\left(\mathbf{x}^{T} B_{\rho} \mathbf{x}\right)^{\frac{1}{2}},
\end{aligned}
$$

for all $\mathbf{x} \in \mathbb{R}^{\nu+1}$ and $\rho$ sufficiently small. We define the Lyapunov functional

$$
V_{\rho}(t, \mathbf{x}(\cdot))=\left(\mathbf{x}^{T} B_{\rho} \mathbf{x}\right)^{\frac{1}{2}}+\bar{K} \int_{0}^{t} \int_{t}^{\frac{1}{\rho}}\left\|D_{\rho}(\xi-s)\right\| d \xi|\mathbf{x}(s)| d s
$$


with some $\bar{K}>0$ chosen independently of $\rho$. Following the calculations in [2, p. 38] we find for the derivative of $V_{\rho}$ along solutions to (3.31)

$$
\begin{aligned}
\frac{d}{d t}\left[V_{\rho}\left(t, \mathbf{z}_{\rho}(t)\right)\right] \leq & -\left(L-\bar{K} \int_{t}^{\frac{1}{\rho}}\left\|D_{\rho}(\xi-t)\right\| d \xi\right)\left|\mathbf{z}_{\rho}(t)\right| \\
& -(\bar{K}-K) \int_{0}^{t}\left\|D_{\rho}(t-s)\right\|\left|\mathbf{z}_{\rho}(s)\right| d s
\end{aligned}
$$

We have

$$
\begin{aligned}
\int_{t}^{\frac{1}{\rho}}\left\|D_{\rho}(\xi-t)\right\| d \xi & \leq \frac{\rho^{\nu+2}}{c} \int_{t}^{\frac{1}{\rho}}\left|\tilde{k}^{(\nu)}(\rho(\xi-t))\right| d \xi \\
& =\frac{\rho^{\nu+1}}{c} \int_{0}^{1-\rho t}\left|\tilde{k}^{(\nu)}(s)\right| d \\
& \leq \frac{\rho^{\nu+1}}{c} \int_{0}^{\rho} \lambda(\tau) \int_{0}^{1-\rho t}\left|k^{(\nu)}(s+\tau)\right| d s d \omega(\tau) \\
& \leq\left\|k^{(\nu)}\right\|_{L^{1}(0,1+\rho)}\left(\frac{(\nu+1) !}{k_{\nu}} \frac{C_{\nu+1}}{C_{2 \nu+2}}+o(\rho)\right) .
\end{aligned}
$$

Here we used (3.5) and (3.8). We want to choose $\bar{K}$ in such a way that $\bar{K}-K \geq 0$ and there exists $\bar{L}>0$ such that

$$
\left(L-\bar{K} \int_{t}^{\frac{1}{\rho}}\left\|D_{\rho}(\xi-t)\right\| d \xi\right) \geq \bar{L}
$$

for $\rho$ sufficiently small and for all $t \in\left[1, \frac{1}{\rho}\right]$. Using the estimate (3.42) we see that $\bar{K}$ can be chosen satisfying the above inequalities if

$$
K \leq \bar{K} \leq \frac{(L-\bar{L}) k_{\nu} C_{2 \nu+2}}{(\nu+1) !\left\|k^{(\nu)}\right\|_{L^{1}(0,1+\rho)} C_{\nu+1}}+o(\rho) .
$$

Now it is easily seen that we can find appropriate $\bar{L}$ and $\bar{K}$ if we have

$$
\frac{\left\|k^{(\nu)}\right\|_{L^{1}(0,1)}}{k_{\nu}}<\frac{L C_{2 \nu+2}}{K(\nu+1) ! C_{\nu+1}}=\tilde{C} .
$$

This is the constant $\tilde{C}$ referred to in the formulation of the lemma. Note that $\tilde{C}$ depends exclusively on the $C_{l}$ 's and hence only on the choice of $\omega$. With (3.43) and $\bar{K}-K \geq 0$ we conclude from (3.41) that

$$
\frac{d}{d t}\left[V_{\rho}\left(t, \mathbf{z}_{\rho}(t)\right)\right] \leq-\bar{L}\left|\mathbf{z}_{\rho}(t)\right| .
$$

Integrating this relation yields

$$
V_{\rho}\left(t, \mathbf{z}_{\rho}(t)\right) \leq V_{\rho}\left(0, \mathbf{z}_{\rho}(0)\right)-\bar{L} \int_{0}^{t}\left|\mathbf{z}_{\rho}(s)\right| d s .
$$

With (3.40), (3.37), and (3.39) we obtain

$$
\frac{1}{R}\left|\mathbf{z}_{\rho}(t)\right| \leq \frac{1}{2 L}\left|\mathbf{z}_{\rho}(0)\right|-\bar{L} \int_{0}^{t}\left|\mathbf{z}_{\rho}(s)\right| d s,
$$


and using Gronwall's inequality we find

$$
\left|\mathbf{z}_{\rho}(t)\right| \leq \frac{R}{2 L} e^{-R \bar{L} t}\left|\mathbf{z}_{\rho}(0)\right|
$$

and

$$
\left\|\mathbf{z}_{\rho}\right\|_{L^{1}\left(0, \frac{1}{\rho}\right)} \leq \frac{1}{2 L \bar{L}}\left|\mathbf{z}_{\rho}(0)\right| .
$$

Therefore, for the solution $z_{\rho}$ of the homogeneous problem (3.24) with initial condition $z_{\rho}(0)=1$ we obtain

$$
\left|z_{\rho}(t)\right| \leq\left|\mathbf{z}_{\rho}(t)\right| \leq \frac{R d}{2 L}
$$

and

$$
\left\|z_{\rho}\right\|_{L^{1}\left(0, \frac{1}{\rho}\right)} \leq\left\|\mathbf{z}_{\rho}\right\|_{L^{1}\left(0, \frac{1}{\rho}\right)} \leq \frac{d}{2 L \bar{L}}
$$

by (3.35) for all $\rho$ sufficiently small. Setting

$$
m=\max \left(\frac{R d}{2 L}, \frac{d}{2 L \bar{L}}\right)
$$

proves the claim.

For the stability estimates in Proposition 3.1 we did not need condition $\left(A_{3}\right)$. With $\left(\mathrm{A}_{3}\right)$ holding and with Lemma 3.2 we can get a much better estimate for $\|u\|_{\mathcal{C}[0,1]}$.

TheOREm 3.3. Let $f$ be continuous on $[0,1+R)$ for some $R>0$, and let $k$ be $\nu$-smoothing with $\nu>0$. Let $a, b$, and $c$ be given by (2.18), and suppose that $\tilde{g}$, $\tilde{f}$ and $\tilde{k}$ are defined by (2.19), (2.20), and (2.21), respectively. Assume, moreover, that assumptions $\left(\mathrm{A}_{1}\right),\left(\mathrm{A}_{2}\right)$, and $\left(\mathrm{A}_{3}\right)$ hold. With $\tilde{C}$ denoting the constant in Lemma 3.2 and $u$ denoting the solution to (2.23) we have

$$
|u(t)| \leq \frac{\bar{m}}{\rho^{\nu}}\|f\|_{\mathcal{C}[0,1+\rho]} \quad \text { for all } t \in[0,1]
$$

for some constant $\bar{m}>0$ independent of $\rho$ provided that

$$
\frac{\left\|k^{(\nu)}\right\|_{L^{1}(0,1)}}{k_{\nu}}<\tilde{C}
$$

holds.

Proof. By setting $y(t)=u(\rho t)$ we get

$$
y^{\prime}(t)=-\frac{b \rho}{c} y(t)-\frac{\rho^{2}}{c} \int_{0}^{t} \tilde{k}(\rho(t-s)) y(s) d s+G(t)
$$

on $\left[0, \frac{1}{\rho}\right]$ with

$$
G(t)=\frac{\rho}{c} \int_{0}^{\rho} \lambda(\tau) f(\rho t+\tau) d \omega(\tau)
$$


Using (3.5) and (3.8) we get the estimate

$$
|G(t)| \leq \frac{1}{\rho^{\nu}}\left(\frac{(\nu+1) ! C_{\nu}}{C_{2 \nu+2} k_{\nu}}+o(\rho)\right)\|f\|_{\mathcal{C}[0,1+\rho]}
$$

on $\left[0, \frac{1}{\rho}\right]$ for $\rho$ sufficiently small.

Now we can use the variation of constant formula (see [2, Thm. 2.3.1, p. 29]) to express $y$. We have

$$
y(t)=z_{\rho}(t) y(0)+\int_{0}^{t} z_{\rho}(t-s) G(s) d s .
$$

Hence, using Lemma 3.2 together with estimate (3.14) in the proof of Proposition 3.1 and (3.48) (note that $y(0)=u(0)$ ) we obtain

$$
\begin{aligned}
|y(t)| & \leq\left|z_{\rho}(t)\right||y(0)|+\left\|z_{\rho}\right\|_{L^{1}\left(0, \frac{1}{\rho}\right)}\|G\|_{\mathcal{C}\left[0, \frac{1}{\rho}\right]} \\
& \leq \frac{m}{\rho^{\nu}}\left(\frac{\nu !}{k_{\nu}} \frac{C_{2 \nu+2} C_{\nu}+C_{2 \nu+1} C_{\nu+1}}{C_{2 \nu+2} C_{2 \nu}-C_{2 \nu+1}^{2}}+\frac{(\nu+1) ! C_{\nu}}{C_{2 \nu+2} k_{\nu}}+o(\rho)\right)\|f\|_{\mathcal{C}[0,1+\rho]} \\
& \leq \frac{\bar{m}}{\rho^{\nu}}\|f\|_{\mathcal{C}[0,1+\rho]}
\end{aligned}
$$

for some constant $\bar{m}>0$ independent of $\rho$. Since $u(t)=y\left(\frac{t}{\rho}\right)$, the above estimate also holds for $u$.

4. Convergence analysis. Suppose that we are given functions $f_{0}$ and $u_{0}$ which are continuous on $[0,1+R)$ and which satisfy

$$
\int_{0}^{t} k(t-s) u_{0}(s) d s=f_{0}(t) \text { on }[0,1+R)
$$

We refer to $u_{0}$ as the exact solution to (4.1) with attainable data $f_{0}$. Under the assumption (2.9) that $k$ is $\nu$-smoothing, it is seen that

$$
\begin{aligned}
& f_{0}^{(l)}(t)=\int_{0}^{t} k^{(l)}(t-s) u_{0}(s) d s \quad \text { for } l=0, \ldots, \nu-1, \\
& f_{0}^{(\nu)}(t)=k_{\nu} u_{0}(t)+\int_{0}^{t} k^{(\nu)}(t-s) u_{0}(s) d s .
\end{aligned}
$$

Thus, we have $f_{0} \in \mathcal{C}^{(\nu)}[0,1+\rho]$ for all $\rho<R$ and $f_{0}^{(l)}(0)=0$ for $l=0, \ldots, \nu-1$ and

$$
f_{0}^{(\nu)}(0)=k_{\nu} u_{0}(0)
$$

Let $\tilde{g}_{0}$ and $\tilde{f}_{0}$ be constructed from $f_{0}$ by $(2.19)$ and $(2.20)$, respectively. We can use (4.2) to derive the asymptotic expansions

$$
\begin{aligned}
\tilde{f}_{0}(0) & =\rho^{s+2 \nu+1}\left(u_{0}(0) \frac{k_{\nu}^{2}}{\nu !(\nu+1) !} C_{2 \nu+1}+o(\rho)\right), \\
\tilde{g}_{0} & =\rho^{s+2 \nu}\left(u_{0}(0) \frac{k_{\nu}^{2}}{((\nu+1) !)^{2}} C_{2 \nu}+o(\rho)\right) .
\end{aligned}
$$

We have the following convergence result for the approximating regularization scheme (2.23). 
THEOREM 4.1. Let $u_{0}$ and $f_{0}$ be given satisfying (4.1). We assume additionally that $u_{0}$ is continuously differentiable on $[0,1+R)$. Let

$$
\mu\left(u_{0}, \rho\right)=\max _{s, t \in[0,1+\rho],|s-t| \leq \rho}\left(\left|u_{0}^{\prime}(s)-u_{0}^{\prime}(t)\right|\right) .
$$

Furthermore, let $u_{\rho}$ denote the solution to (2.23) with data given by $\tilde{f}_{0}$ and $\tilde{g}_{0}$, and assume that conditions $\left(\mathrm{A}_{1}\right),\left(\mathrm{A}_{2}\right)$, and $\left(\mathrm{A}_{3}\right)$ hold. Then there exist constants $\tilde{C}>0$ (depending on $\omega$ ) and $\bar{M}>0$ (depending on $k$ and $\omega$ ) such that

$$
\left|u_{\rho}(t)-u_{0}(t)\right| \leq \bar{M} \mu\left(u_{0}, \rho\right) \text { for all } t \in[0,1]
$$

whenever

$$
\frac{\left\|k^{(\nu)}\right\|_{L^{1}(0,1)}}{k_{\nu}}<\tilde{C}
$$

Proof. Evaluating (4.1) at the point $t+\tau$, multiplying by $\lambda(\tau)$, and integrating from 0 to $\rho$ with respect to the measure $\omega$ yields

$$
\int_{0}^{\rho} \lambda(\tau) \int_{0}^{t+\tau} k(t+\tau-s) u_{0}(s) d s d \omega(\tau)=\int_{0}^{\rho} \lambda(\tau) f_{0}(t+\tau) d \omega(\tau) .
$$

Hence,

$$
\begin{aligned}
\int_{s=0}^{t} & \left(\int_{\tau=0}^{\rho} \lambda(\tau) k(t-s+\tau) d \omega(\tau)\right) u_{0}(s) d s \\
& +\int_{\tau=0}^{\rho} \lambda(\tau)\left(\int_{s=t}^{t+\tau} k(t-s+\tau) u_{0}(s) d s\right) d \omega(\tau)=\tilde{f}_{0}(t) .
\end{aligned}
$$

Since $u_{0}$ is continuously differentiable we have

$$
u_{0}(s)=u_{0}(t)+u_{0}^{\prime}(t)(s-t)+\int_{t}^{s}\left(u_{0}^{\prime}(\xi)-u_{0}^{\prime}(t)\right) d \xi .
$$

With this we find

$$
\begin{aligned}
\int_{0}^{\rho} \lambda(\tau) & \left(\int_{s=t}^{t+\tau} k(t-s+\tau) u_{0}(s) d s\right) d \omega(\tau) \\
= & u_{0}(t) \int_{0}^{\rho} \lambda(\tau)\left(\int_{0}^{\tau} k(s) d s\right) d \omega(\tau) \\
& +u_{0}^{\prime}(t) \int_{0}^{\rho} \lambda(\tau)\left(\int_{0}^{\tau} k(s)(\tau-s) d s\right) d \omega(\tau) \\
& +\int_{0}^{\rho} \lambda(\tau)\left(\int_{0}^{\tau} k(\tau-s)\left(\int_{t}^{t+s}\left(u_{0}^{\prime}(\xi)-u_{0}^{\prime}(t)\right) d \xi\right) d z\right) d \omega(\tau) .
\end{aligned}
$$

With (4.7), and the notation in (2.16)-(2.21), we obtain

$$
\begin{aligned}
& \int_{0}^{t} \tilde{k}(t-s) u_{0}(s) d s+b u_{0}(t)+c u_{0}^{\prime}(t) \\
& \quad=\tilde{f}_{0}(t)-\int_{0}^{\rho} \lambda(\tau)\left(\int_{0}^{\tau} k(\tau-s)\left(\int_{t}^{t+s}\left(u_{0}^{\prime}(\xi)-u_{0}^{\prime}(t)\right) d \xi\right) d s\right) d \omega(\tau) .
\end{aligned}
$$


We set $v_{\rho}=u_{\rho}-u_{0}$. Since $u_{\rho}$ is the solution to (2.23b) with the right-hand side given by $\tilde{f}_{0}$ we obtain

$$
\begin{aligned}
& \int_{0}^{t} \tilde{k}(t-s) v_{\rho}(s) d s+b v_{\rho}(t)+c v_{\rho}^{\prime}(t) \\
& \quad=\int_{0}^{\rho} \lambda(\tau)\left(\int_{0}^{\tau} k(\tau-s)\left(\int_{t}^{t+s}\left(u_{0}^{\prime}(\xi)-u_{0}^{\prime}(t)\right) d \xi\right) d s\right) d \omega(\tau) .
\end{aligned}
$$

Before we investigate (4.9) more closely we estimate the initial value $v_{\rho}(0)$. Solving $(2.23 \mathrm{a})$ for $u_{\rho}(0)$ gives

$$
u_{\rho}(0)=\frac{c \tilde{g}_{0}-b \tilde{f}_{0}(0)}{a c-b^{2}} .
$$

Hence, we have

$$
\begin{aligned}
\left|u_{\rho}(0)-u_{0}(0)\right| & =\left|\frac{c \tilde{g}_{0}-b \tilde{f}_{0}(0)}{a c-b^{2}}-\frac{a c-b^{2}}{a c-b^{2}} u_{0}(0)\right| \\
& \leq \frac{c}{a c-b^{2}}\left|\tilde{g}_{0}-a u_{0}(0)\right|+\frac{b}{a c-b^{2}}\left|\tilde{f}_{0}(0)-b u_{0}(0)\right| .
\end{aligned}
$$

Using (2.19), (2.18), and $f_{0}(\tau)=\int_{0}^{\tau} k(s) u_{0}(\tau-s) d s$, we find

$$
\begin{aligned}
\left|\tilde{g}_{0}-a u_{0}(0)\right| & =\left|\int_{0}^{\rho} \kappa(\tau) \int_{0}^{\tau} k(s)\left(u_{0}(\tau-s)-u_{0}(0)\right) d s d \omega(\tau)\right| \\
& \leq a \max _{\xi \in[0, \rho]}\left(\left|u_{0}(\xi)-u_{0}(0)\right|\right) \leq a \rho\left\|u_{0}^{\prime}\right\|_{\mathcal{C}[0,1+\rho]} .
\end{aligned}
$$

Analogously we obtain

$$
\left|\tilde{f}_{0}(0)-b u_{0}(0)\right| \leq b \rho\left\|u_{0}^{\prime}\right\|_{\mathcal{C}[0,1+\rho]} .
$$

Thus, we have

$$
\left|v_{\rho}(0)\right| \leq \frac{a c+b^{2}}{a c-b^{2}} \rho\left\|u_{0}^{\prime}\right\|_{\mathcal{C}[0,1+\rho]} .
$$

If we use the asymptotic expressions (3.6)-(3.8) for $a, b$, and $c$, respectively, and assumption $\left(\mathrm{A}_{2}\right)$ it is easy to see that there exists a constant $n_{1}>0$ independent of $\rho$ such that

$$
\left|v_{\rho}(0)\right| \leq n_{1} \rho .
$$

Now we come back to the analysis of (4.9). With the transformation $y_{\rho}(t)=v_{\rho}(\rho t)$ we obtain from (4.9)

$$
y_{\rho}^{\prime}(t)=-\frac{b \rho}{c} y_{\rho}(t)-\frac{\rho^{2}}{c} \int_{0}^{t} \tilde{k}(\rho(t-s)) y_{\rho}(s) d s+F_{\rho}(t)
$$

for $t \in\left[0, \frac{1}{\rho}\right]$ with

$$
F_{\rho}(t)=\frac{1}{c} \int_{0}^{\rho} \lambda(\tau)\left(\int_{0}^{\tau} k(\tau-s)\left(\int_{\rho t}^{\rho t+s}\left(u_{0}^{\prime}(\xi)-u_{0}^{\prime}(\rho t)\right) d \xi\right) d s\right) d \omega(\tau) .
$$


We have the following estimate for $F_{\rho}$ :

$$
\begin{aligned}
\left|F_{\rho}(t)\right| & \leq \max _{\xi \in[\rho t, \rho t+\rho]}\left|u_{0}^{\prime}(\xi)-u_{0}^{\prime}(\rho t)\right| \frac{1}{c} \int_{0}^{\rho} \lambda(\tau) \int_{0}^{\tau}(\tau-s) k(s) d s d \omega(\tau) \\
& =\max _{\xi \in[\rho t, \rho t+\rho]}\left|u_{0}^{\prime}(\xi)-u_{0}^{\prime}(\rho t)\right| \leq \mu\left(u_{0}, \rho\right)
\end{aligned}
$$

for all $t \in\left[0, \frac{1}{\rho}\right]$.

Now we can use the fundamental solution $z_{\rho}$ for (3.24) and the variation of constants formula (see [2, Thm. 2.3.1, p. 29]) to obtain an estimate for the solution of the inhomogeneous problem (4.11). We have

$$
y_{\rho}(t)=z_{\rho}(t) y_{\rho}(0)+\int_{0}^{t} z_{\rho}(t-s) F_{\rho}(s) d s .
$$

Consequently, using the estimates in Lemma 3.2 we find

$$
\begin{aligned}
\left|y_{\rho}(t)\right| & \leq\left|z_{\rho}(t)\right|\left|y_{\rho}(0)\right|+\left\|z_{\rho}\right\|_{L^{1}\left(0, \frac{1}{\rho}\right)}\left\|F_{\rho}\right\|_{\mathcal{C}\left[0, \frac{1}{\rho}\right]} \\
& \leq \bar{m}\left(\left|y_{\rho}(0)\right|+\left\|F_{\rho}\right\|_{\mathcal{C}\left[0, \frac{1}{\rho}\right]}\right) .
\end{aligned}
$$

We can use $(4.10),(4.13)$, and the fact that $y_{\rho}(0)=v_{\rho}(0)$ to conclude that

$$
\left|y_{\rho}(t)\right| \leq \bar{m}\left(n_{1} \rho+\mu\left(u_{0}, \rho\right)\right) \leq \bar{M} \mu\left(u_{0}, \rho\right)
$$

for some constant $\bar{M}>0$ independent of $\rho$ and for all $t \in\left[0, \frac{1}{\rho}\right]$. With $\left|v_{\rho}(t)\right|=\left|y_{\rho}\left(\frac{t}{\rho}\right)\right|$ the proof is complete.

Remark 4 . Since $u_{0}^{\prime}$ is uniformly continuous on $[0,1+\rho]$, it is clear that $\mu\left(u_{0}, \rho\right) \rightarrow$ 0 as $\rho \rightarrow 0$. If the exact solution $u_{0} \in \mathcal{C}^{1, \alpha}[0,1+\rho]$, that is, the first derivative is Hölder continuous of order $0<\alpha \leq 1$, then we have $\mu\left(u_{0}, \rho\right)=O\left(\rho^{\alpha}\right)$ and we get the estimate

$$
\left|u_{\rho}(t)-u_{0}(t)\right| \leq m \rho^{\alpha} .
$$

Remark 5. In the above proof we considered stability properties of the homogeneous system (3.31) and we used the variation of constants formula to estimate $v_{\rho}$. In the convergence proof for the corresponding zero-order method in [6] the inhomogeneous problem is transformed into a system by differentiation, thus yielding the requirement that the exact solution $u_{0}$ must be $\mathcal{C}^{\nu}$-smooth. By our method of proof we could avoid such high regularity assumptions on $u_{0}$.

In the case of noisy data we have the following convergence result.

TheOREM 4.2. Suppose the notation and conditions of Theorems 3.3 and 4.1 hold. Let a family of functions $\left\{f_{\delta}\right\}_{\delta>0} \subset \mathcal{C}\left[0,1+\rho_{0}\right]$ be given satisfying

$$
\left|f_{\delta}(t)-f_{0}(t)\right| \leq \delta
$$

for all $t \in\left[0,1+\rho_{0}\right]$ with some $\rho_{0}>0$. Moreover, denote by $u_{\rho}^{\delta}$ the solution to $(2.23)$ with data $\tilde{f}_{\delta}$ and $\tilde{g}_{\delta}$ defined by means of $f=f_{\delta}$. Then there exists $\rho=\rho(\delta)$ such that

$$
\left|u_{\rho(\delta)}^{\delta}(t)-u_{0}(t)\right| \rightarrow 0 \quad \text { as } \delta \rightarrow 0
$$

uniformly in $t \in[0,1]$. 
If additionally $u_{0} \in \mathcal{C}^{1, \alpha}\left[0,1+\rho_{0}\right]$ for some $0<\alpha \leq 1$, then there exists a constant $M>0$ such that for the choice

$$
\rho(\delta)=\delta^{\frac{1}{\alpha+\nu}}
$$

we have

$$
\left|u_{\rho(\delta)}^{\delta}(t)-u_{0}(t)\right| \leq M \delta^{\frac{\alpha}{\alpha+\nu}}
$$

for all $t \in[0,1]$.

Proof. Using Theorem 3.3 and Theorem 4.1 we get

$$
\begin{aligned}
\left|u_{\rho}^{\delta}(t)-u_{0}(t)\right| & \leq\left|u_{\rho}^{\delta}(t)-u_{\rho}(t)\right|+\left|u_{\rho}(t)-u_{0}(t)\right| \\
& \leq \frac{\bar{m}}{\rho^{\nu}} \delta+\bar{M} \mu\left(u_{0}, \rho\right) .
\end{aligned}
$$

For the choice $\rho(\delta)=\delta^{\frac{1}{2 \nu}}$ we obtain $\mu\left(u_{0}, \rho(\delta)\right) \rightarrow 0$ and $\rho(\delta)^{-\nu} \delta \rightarrow 0$ as $\delta \rightarrow 0$. This proves the first assertion.

If $u_{0}^{\prime}$ is Hölder continuous with exponent $\alpha$ we have $\mu\left(u_{0}, \rho\right) \leq l \rho^{\alpha}$ with some constant $l>0$. Thus, we have

$$
\left|u_{\rho}^{\delta}(t)-u_{0}(t)\right| \leq \frac{\bar{m}}{\rho^{\nu}} \delta+\bar{M} l \rho^{\alpha} \leq \tilde{M}\left(\frac{\delta}{\rho^{\nu}}+\rho^{\alpha}\right),
$$

where $\tilde{M}=\max (\bar{m}, \bar{M} l)$. If we set $\rho(\delta)=\delta^{\frac{1}{\alpha+\nu}}$ we get

$$
\left|u_{\rho(\delta)}^{\delta}(t)-u_{0}(t)\right| \leq 2 \tilde{M} \delta^{\frac{\alpha}{\alpha+\nu}}
$$

for all $t \in[0,1]$. Setting $M=2 \tilde{M}$ completes the proof.

5. Numerical experiments. For all presented numerical examples we use $\omega_{l}=$ 1 for $l=0, \ldots, r$ (uniform weights). Experiments which were carried out with nonuniform weights gave similar results provided that the stability condition $\left(\mathrm{A}_{3}\right)$ is satisfied. The plots in Figure 5.1 show results for the first-order sequential method for different noise levels in the data. We chose $k(t)=t^{2}$, i.e., $k$ is 3 -smoothing. The data are given by $f_{0}(t)=t^{3} / 3-t^{5} / 30$. To the data we added artificial $0-1$ distributed Gaussian noise scaled with a factor $\sigma\|f\|_{\infty}$. The plots in Figure 5.1 show the results for $\sigma=0, \sigma=0.005, \sigma=0.01$, and $\sigma=0.02$. The lengths of the future intervals are $\rho=0.05, \rho=0.4, \rho=0.5$, and $\rho=0.55$, respectively. We used $N=600$ time-steps on the time-interval $[0,3]$. For all numerical experiments presented in this section, the number of time-steps $N$ was chosen relatively large. By doing so we can capture the effects coming from the infinite dimensional regularization strategy (2.23) and keep the superposed effect of regularization by discretization rather small. The plots show the exact solution as dashed lines and the reconstructed solutions from noisy data as solid lines. It is seen that the first-order method gives stable results in the presence of data noise.

The plots in Figure 5.2 show a comparison between first- and zero-order sequential regularization, Tikhonov regularization, and the Cinzori-Lamm first-order polynomial method described in [3]. We used again the kernel $k(t)=t^{2}(\nu=3)$, but with different data $f_{0}(t)=-1 / 32 \sin 4 t-1 / 8 t$ for $t \in[0,3]$. With these data there is more variation in the exact solution $u_{0}$ which makes it more difficult to reconstruct. 

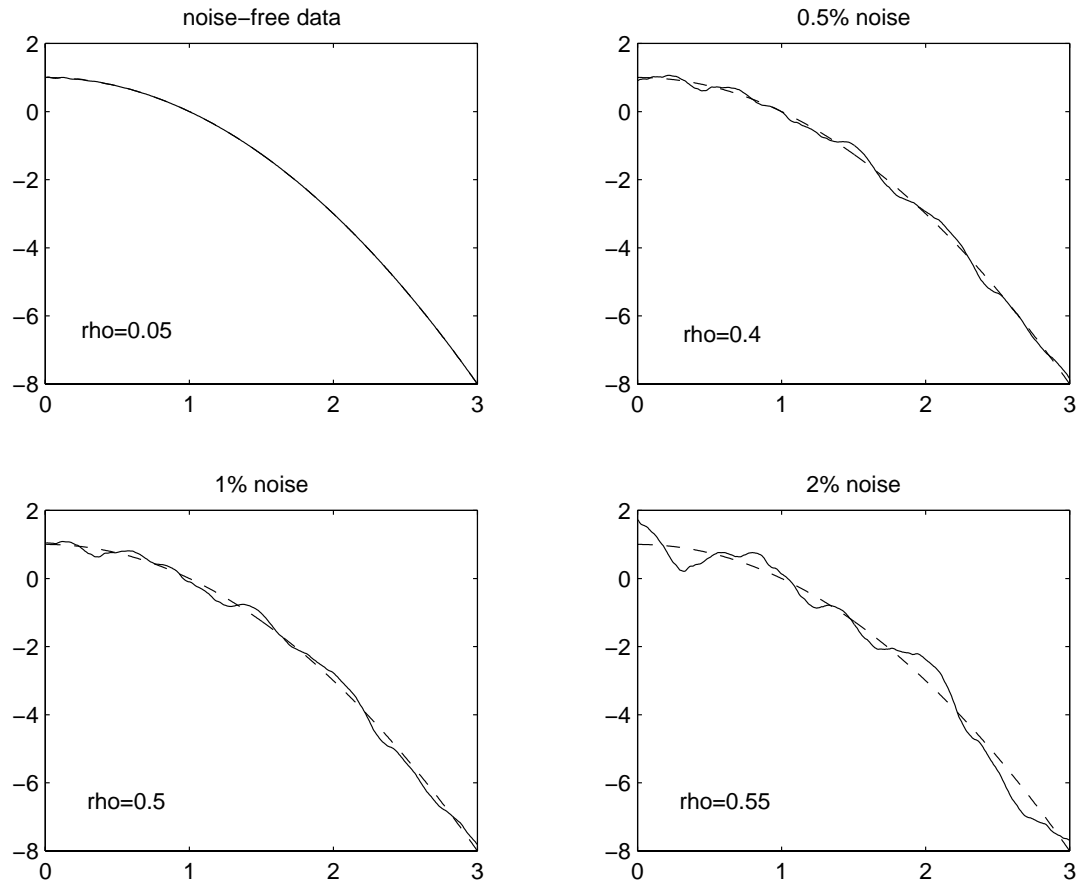

FIG. 5.1. Solutions for first-order sequential regularization with different noise levels.

The noise level is $2 \%$. We used $N=600$ collocation points and $r=80$ future points which means $\rho=0.4$ for the length of the future interval, both for the firstand for the zero-order method. Both lengths were chosen experimentally to give optimal $L^{2}$-errors for the reconstruction. The fact that the optimal regularization parameter is (approximately) given by $\rho=0.4$ in both cases is mere accident. The same strategy was pursued to find the "optimal" regularization parameter for the Tikhonov method. It is apparent that the first-order method produces smoother results than the zero-order method with comparable supremum-norm errors. On most of the considered time-intervals Tikhonov's method performs better than the sequential methods. The poor performance of Tikhonov's method in the last part of the time-interval can be attributed to the fact that our implementation of the Tikhonov functional does not make use of data outside the interval $[0,3]$, whereas the sequential methods make use of data up to time $t=3.4$. What is seen in the last part of the interval is, therefore, not a regularization phenomenon but rather an inherent difficulty of Volterra inverse problems. For the chosen discretization (600 time-steps) computation by the first-order method is faster by a factor 10 ( 2.3 seconds versus 21.4 seconds) when compared to Tikhonov's method. Moreover, if further data points are added, the already computed first-order solution can be extended with minimal numerical effort. For Tikhonov regularization the whole system matrix has to be reassambled and the solution has to be recomputed from the initial time zero onwards. The last graph in Figure 5.2 shows the results for a first-order CinzoriLamm method (see section 1 or [3] for a brief description). The optimal length for the future interval is $\rho=0.8$ in this case. Therefore, we need twice as much information from the future than as for the other sequential methods. The $L^{2}$-norm of the error is slightly better, but the solution is less smooth than the solution obtained by the first-order sequential method. 

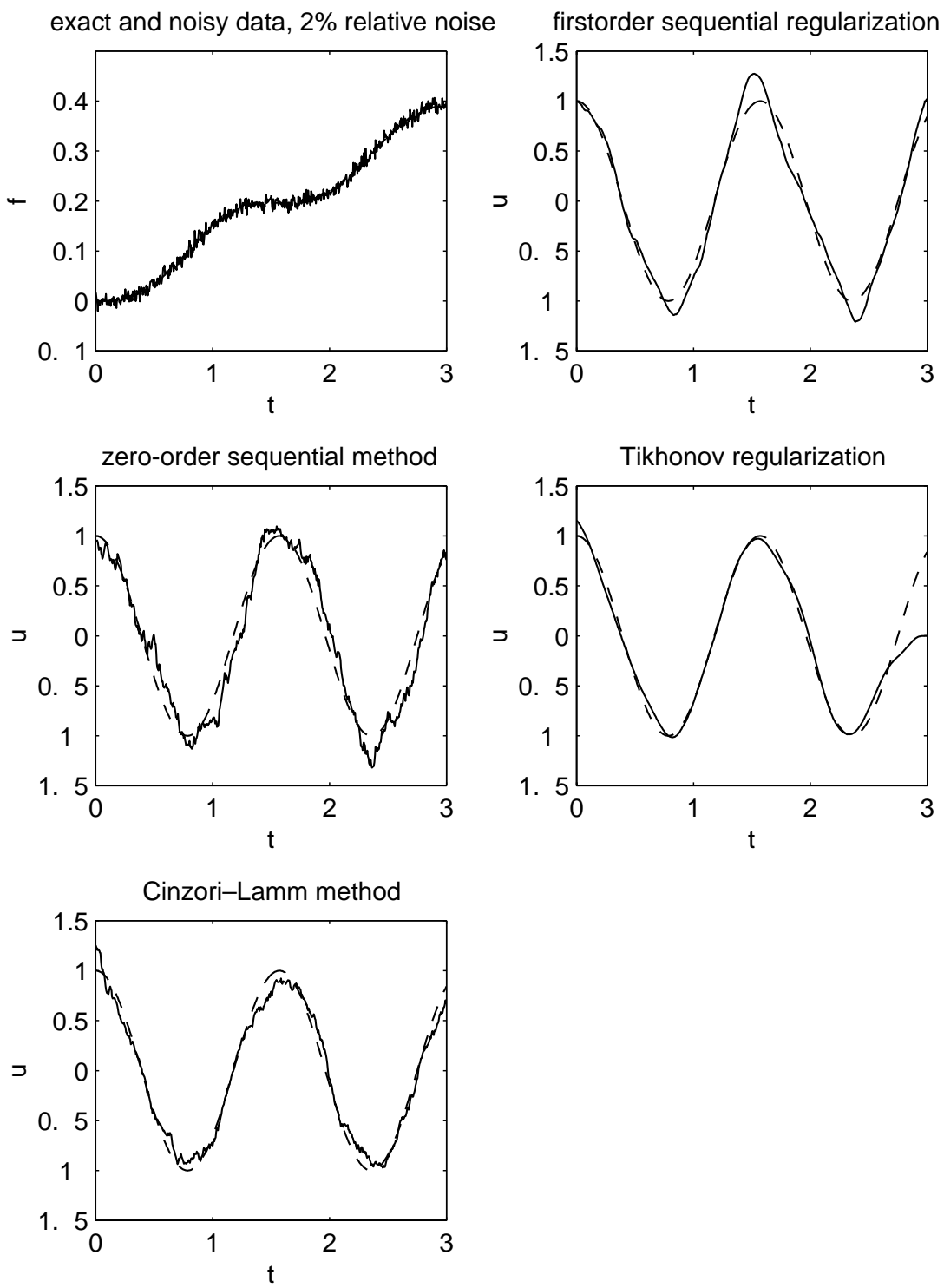

FIG. 5.2. Comparison between different regularization methods for noisy data.

In Figure 5.3 we compare first- and zero-order sequential regularization for the case that the future interval is chosen too large. With the same specifications as in Figure 5.2, but with $\rho=0.9$, we see that the zero-order method over-regularizes strongly, resulting in a strongly damped but still not very smooth solution. On the other hand, the first-order method produces a solution with a qualitatively correct amplitude but with a delayed phase.

In Figure 5.4 the stars $(*)$ and circles $(\circ)$ show the locations of the roots of the polynomial

$$
\sum_{l=0}^{\nu+1} \frac{C_{\nu+l+1}}{l !} x^{l}
$$



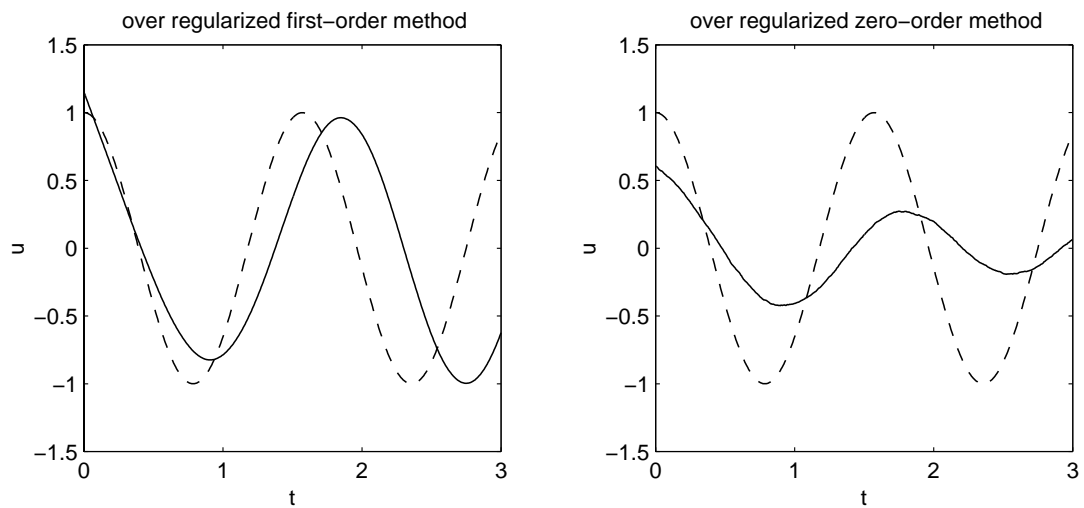

FIG. 5.3. Over-regularization for first- and zero-order method.
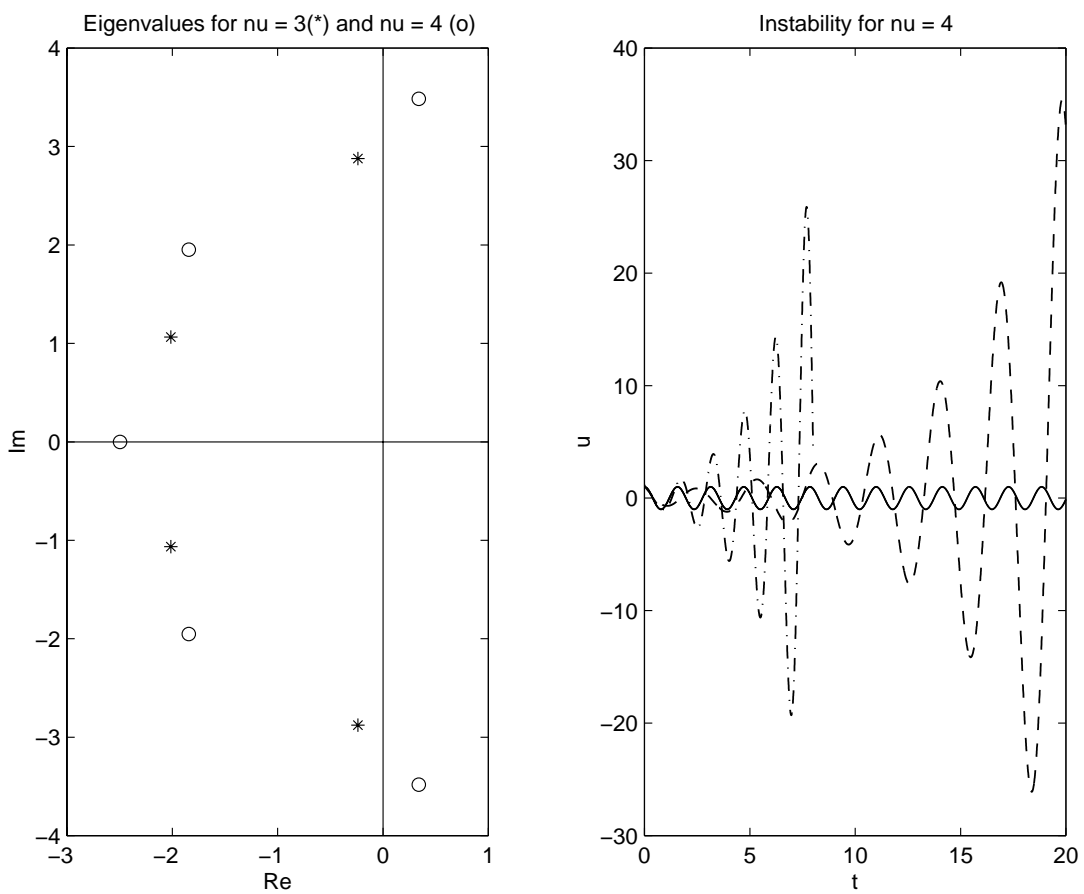

FIG. 5.4. Location of roots of the polynomial (5.1) for $\nu=3(*)$ and $\nu=4(0)$. Solutions for $\nu=4$ in case of noise-free data with different values of $\rho$.

for $\nu=3$ and $\nu=4$, respectively, in the complex plane for the uniform weight $\omega \equiv 1$. It is seen that for $\nu=3$, all roots have negative real parts. For $\nu=4$ one pair of roots has crossed the imaginary axis, implying that assumption $\left(\mathrm{A}_{3}\right)$ is not satisfied in this case. The instability effect of condition $\left(\mathrm{A}_{3}\right)$ not being satisfied is also indicated in Figure 5.4. Here we chose $k(t)=t^{3}$ (i.e., $\nu=4$ ) with no noise on the data. We used two different (rather large) values $\rho=0.8$ (dash-dotted line) and $\rho=1.6$ (dashed line) for the length of the future interval. The exact solution is given by the solid line. It is seen that for both values of $\rho$ the solution of the first-order method behaves like an unstable oscillator. For the larger value of $\rho$ the blow-up of the solution occurs at a 
later time, but the error in the phase of the oscillation is much larger for the bigger value of $\rho$. The instability is actually an inherent property of the sequential method and not only a result of under-regularization. It occurs for very large future intervals and for noise-free data.

For $n=4$, a sophisticated construction gives a stabilizing weight $\omega$ for the zeroorder method (see [6]). It has been recently shown in [10] that a stability condition corresponding to $\left(\mathrm{A}_{3}\right)$ for the zero-order sequential method is never satisfied for any given weight $\omega$ if $\nu \geq 5$. The construction of a stable sequential regularization method for $\nu$-smoothing kernels with $\nu \geq 5$ is still an open problem.

6. Conclusions. Starting with Beck's linear function specification method we found a family of approximating Volterra integro-differential equations of the second kind for the stable solution of ill-posed Volterra equations of the first kind. We proved stability estimates and convergence if the data-noise goes to zero. A convergence rate depending on smoothness properties of the exact solution was derived. Numerical experiments showed that the method produces smoother results than the well investigated sequential predictor-corrector method. The second-kind Volterra integrodifferential equation can be solved sequentially in time, which makes the algorithm much faster than, for example, Tikhonov regularization.

Acknowledgments. The author thanks Karl Kunisch and Stephen Keeling for their valuable comments.

\section{REFERENCES}

[1] J. V. Beck, B. Blackwell, and C. R. St. Clair, Inverse Heat Conduction; Ill-Posed Problems, Wiley Interscience, New York, Chichester, Brisbane, 1985.

[2] T. A. Burton, Volterra Integral and Differential Equations, Math. Sci. Engrg. 167, Academic Press, New York, 1983.

[3] A. C. Cinzori and P. K. Lamm, Future polynomial regularization of ill-posed Volterra equations, SIAM J. Numer. Anal., 37 (2000), pp. 949-979.

[4] P. K. Lamm, Future-sequential regularization methods for ill-posed Volterra equations, J. Math. Anal. Appl., 195 (1995), pp. 469-494.

[5] P. K. LAMm, Approximation of ill-posed Volterra problems via predictor-corrector regularization methods, SIAM J. Appl. Math., 56 (1996), pp. 524-541.

[6] P. K. Lamm, Regularized inversion of finitely smoothing Volterra operators: Predictor-corrector regularization methods, Inverse Problems, 13 (1997), pp. 375-402.

[7] P. K. Lamm, A survey of regularization methods for first-kind Volterra equations, in Surveys on Solution Methods for Inverse Problems, D. Colton, H. W. Engl, A. Louis, J. R. McLaughlin, and W. Rundell, eds., Springer, Vienna, 2000, pp. 53-82.

[8] P. K. Lamm and T. L. Scofield, Local Regularization Methods for the Stabilization of IllPosed Volterra Problems, Preprint, Department of Mathematics, Michigan State University, East Lansing, 2000.

[9] P. K. Lamm And T. L. Scofield, Sequential predictor-corrector methods for the variable regularization of Volterra inverse problems, Inverse Problems, 16 (2000), pp. 373-399.

[10] W. Ring AND J. PRIX, Sequential predictor-corrector regularization methods and their limitations, Inverse Problems, 16 (2000), pp. 619-633. 